\title{
Complete Substrate Inhibition of Cytochrome P450 2C8 by AZD9496, an Oral Selective Estrogen Receptor Degrader
}

\author{
Tashinga E. Bapiro, Andy Sykes, Scott Martin, Michael Davies, James W. T. Yates, Matthias Hoch, \\ Helen E. Rollison, and Barry Jones
}

\begin{abstract}
Oncology (T.E.B., A.S., S.M., M.D., J.W.T.Y., B.J.), Quantitative Clinical Pharmacology, Early Clinical Development (M.H.), and Drug Safety and Metabolism (H.E.R.), IMED Biotech Unit, AstraZeneca, Cambridge, United Kingdom
\end{abstract}

Received March 19, 2018; accepted June 14, 2018

\begin{abstract}
AZD9496 ((E)-3-(3,5-difluoro-4-((1R,3R)-2-(2-fluoro-2-methylpropyl)3-methyl-2,3,4,9-tetrahydro-1H-pyrido[3,4-b]indol-1-yl)phenyl)acrylic acid) is an oral selective estrogen receptor degrader currently in clinical development for treatment of estrogen receptor-positive breast cancer. In a first-in-human phase 1 study, AZD9496 exhibited dose nonlinear pharmacokinetics, the mechanistic basis of which was investigated in this study. The metabolism kinetics of AZD9496 were studied using human liver microsomes (HLMs), recombinant cytochrome P450s (rP450s), and hepatocytes. In addition, modeling approaches were used to gain further mechanistic insights. CYP2C8 was predominantly responsible for biotransformation of AZD9496 to its two main metabolites whose rate of formation with increasing AZD9496 concentrations exhibited complete substrate inhibition in HLM, rCYP2C8, and hepatocytes. Total inhibition by AZD9496 of
\end{abstract}

amodiaquine $\mathrm{N}$-deethylation, a specific probe of CYP2C8 activity, confirmed the completeness of this inhibition. The commonly used substrate inhibition model analogous to uncompetitive inhibition fit poorly to the data. However, using the same model but without constraints on the number of molecules occupying the inhibitory binding site (i.e., $n S_{1} E S$ ) provided a significantly better fit (F test, $P<0.005)$. With the improved model, up to three AZD9496 molecules were predicted to bind the inhibitory site of CYP2C8. In contrast to previous studies showing substrate inhibition of P450s to be partial, our results demonstrate complete substrate inhibition of CYP2C8 via binding of more than one molecule of AZD9496 to the inhibitory site. As CYP2C8 appears to be the sole isoform catalyzing formation of the main metabolites, the substrate inhibition might explain the observed dose nonlinearity in the clinic at higher doses.

\section{Introduction}

The cytochrome P450 (P450) superfamily of enzymes is one of the best-characterized enzyme systems, with a diverse list of substrates that includes over $75 \%$ of currently used drugs whose pharmacokinetics may be impacted by the P450 enzymes (Rendic and Guengerich, 2015). The utility of the drug-P450 interaction knowledge, such as avoidance of drug combinations likely to cause adverse effects due to altered pharmacokinetics, is now well documented (Pelkonen et al., 2005). Indeed, potential drug-P450 interaction studies are an important part of modern drug development programs, and one of the aims of such studies is to provide an understanding of the kinetics of metabolism, which in turn can be used to predict the clearance (Obach et al., 1997; Obach and Reed-Hagen, 2002).

The rates of most $\mathrm{P} 450$-catalyzed reactions can be described by the Michaelis-Menten (M-M) model, in which the rate of product formation increases with increasing substrate concentration to a maximum (Venkatakrishnan et al., 2001). The key kinetic parameters $\mathrm{K}_{\mathrm{m}}$ and $\mathrm{V}_{\max }$ can be determined in vitro (Houston, 1994); however, the data need to be carefully examined to ascertain whether fitting to the M-M equation provides the best estimates of the parameters. For some P450-catalyzed reactions, the apparent maximum rate of product formation is not

Financial support was provided by AstraZeneca.

https://doi.org/10.1124/dmd.118.081539. maintained with further increase in substrate but decreases due to enzyme inhibition by the substrate itself (Spracklin et al., 1997; Tang et al., 2000).

Substrate inhibition of $\mathrm{P} 450 \mathrm{~s}$ is generally considered partial and not complete, as product formation, although reduced, remains measurable with increasing substrate concentration (Tang et al., 2000; Lin et al., 2001). The basis for substrate inhibition of P450s is generally thought to involve binding to a productive and inhibitory site (Korzekwa et al., 1998), with binding to the inhibitory site thought to cause a conformational change that affects binding to the productive site (Williams et al., 2004). For P450s that have large active sites, it is not inconceivable for both sites to be located in close proximity within the active site with possible steric interactions between substrate molecules modifying reaction rates (Lin et al., 2001; Torimoto et al., 2003). Indeed, nonM-M kinetics have been rationalized on the basis of simultaneous binding of multiple ligands within active sites (Atkins, 2005). P450s with large-enough active sites relative to their substrates would likely figure in such interactions and include CYP3A4 and CYP2C8 (Ma et al., 2017).

CYP2C8 is a CYP2C subfamily member that metabolizes about $5 \%$ of therapeutic drugs (Rendic and Guengerich, 2015), with most of the substrates possessing an anionic moiety (Totah and Rettie, 2005). The first crystal structure of $\mathrm{CYP} 2 \mathrm{C} 8$ showed a relatively large active site (Schoch et al., 2004), and additional crystal structures of the enzyme bound to different substrates showed the active site to be T- or Y-shaped

ABBREVIATIONS: AUC, area under the concentration-time curve; AZD9496, (E)-3-(3,5-difluoro-4-((1R,3R)-2-(2-fluoro-2-methylpropyl)-3-methyl2,3,4,9-tetrahydro-1 H-pyrido[3,4-b]indol-1-yl)phenyl)acrylic acid; $\mathrm{CL}_{\text {int }}$, intrinsic clearance; HLM, human liver microsome; $M-M$, Michaelis-Menten; P450, cytochrome P450; rP450, recombinant cytochrome P450. 
A

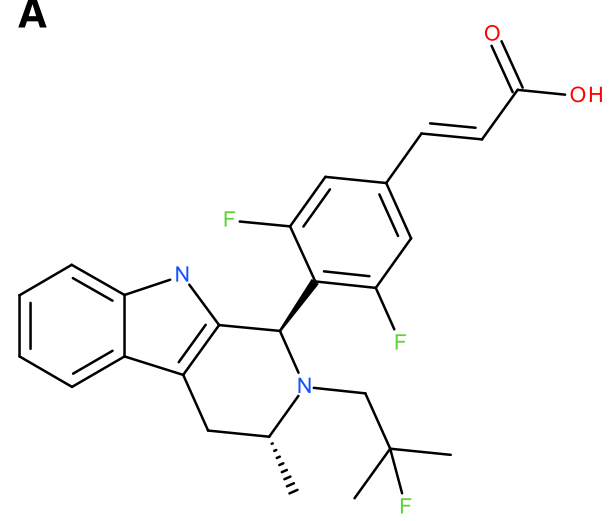

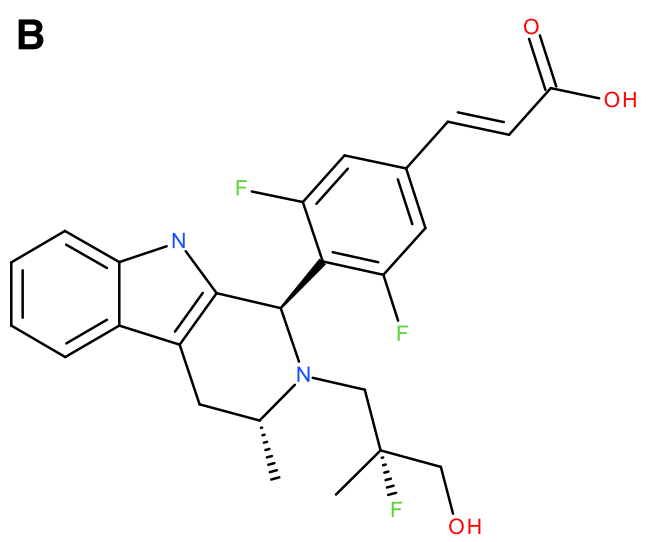

Fig. 1. The chemical structures of AZD 9496 (A) and its metabolites, M3 (the major metabolite) and M5 (minor metabolite) (B); a single structure was used to represent the two metabolites, as they are diastereoisomers but whose configuration is not known. The metabolite standards used in the study were, however, isomerically pure. [reviewed by Gay et al. (2010)]. The size and shape of the CYP2C8 structure seem to allow compounds to adopt different orientations that can impact the rate of metabolism (Ma et al., 2017). Additionally, more than one molecule of a compound can fit in the active site (Schoch et al., 2008), making it possible for interactions involving molecules of the same substrate to modify reaction rates.

AZD9496 (Fig. 1A) is an oral nonsteroidal selective estrogen receptor degrader currently in clinical development for treatment of estrogen receptor-positive breast cancer (De Savi et al., 2015; Weir et al., 2016). A phase 1 study showed AZD9496 to be metabolized to two main metabolites, M3 and M5 (Fig. 1B), detected in plasma (Hamilton et al., 2018). The area under the concentration-time curve (AUC) of AZD9496 increased in reasonable proportion to increasing AZD9496 following single doses from 20 to $400 \mathrm{mg}$. However, a more than dose-proportional increase in AUC and $\mathrm{C}_{\max }$ was observed following a single dose of $600 \mathrm{mg}$. The more than dose-proportional increase in AUC at the single 600 -mg dose was diminished after multiple doses that correlated with an overproportional increase in the ratio of $4 \beta$-hydroxycholesterol to cholesterol. The possible contribution of substrate inhibition to the dose nonlinear pharmacokinetics of AZD9496 was, therefore, investigated in this study.

In contrast to previous reports on substrate inhibition of $\mathrm{P} 450 \mathrm{~s}$ being partial (Lin et al., 2001), complete substrate inhibition of CYP2C8 by AZD9496 was observed in vitro. Modeling of the inhibition kinetics suggested a mechanism that involves binding of at least two molecules of AZD9496 to an inhibitor site. Additionally, amodiaquine deethylation, a high-affinity probe of CYP2C8 activity in vitro (Li et al., 2002), was used to confirm the completeness of the inhibition.

\section{Materials and Methods}

Chemicals and Reagents. AZD9496 and its metabolites M3 and M5 (Fig. 1B) were synthesized in house at AstraZeneca (Cambridge, UK) (De Savi et al., 2015). Amodiaquine hydrochloride, desethylamodiaquine, reduced nicotinamide adenine dinucleotide phosphate (NADPH), and rifampicin were purchased from Sigma-Aldrich (Poole, UK). Cryopreserved human hepatocytes (pool of 10 male and female donors) were purchased from Bioreclamation IVT (Baltimore, MD). Pooled human liver microsomes (HLMs), ultra-pool of 150 male and female donors (catalog no. 452117), were obtained from Corning (Woburn, MA). Male and female individual donor HLMs were obtained from Corning. Recombinant CYP2C8 and control insect cell microsomes were purchased from Corning. All recombinant P450s (rP450s) were obtained from Corning. Methanol and acetonitrile were obtained from Thermo Fisher Scientific (Hemel Hempstead, UK). Lebovitz medium was purchased from Life Technologies (Hemel Hempstead, UK). Cryopreserved HepaRG cells, HepaRG media supplements, CYP2C8-specific primer and probe mix, Dulbecco's phosphate-buffered saline, and Williams' media E were purchased from Thermo Fisher Scientific (Carlsbad,
CA). A FastLane Cell Multiplex NR kit was purchased from Qiagen (Manchester, UK). Glyceraldehyde-3-phosphate dehydrogenase-specific primer and probe mix were purchased from Eurogentec (Liege, Belgium).

Incubation Conditions for Recombinant Human P450s and HLM. The reaction mixture consisted of the appropriate amount of enzyme, potassium phosphate buffer $\mathrm{pH} 7.4$ (final concentration $0.1 \mathrm{M}$ ), NADPH (final concentration $1 \mathrm{mM}$ ), and appropriate concentration of compound (AZD9496 or amodiaquine). After a preincubation period of 5 minutes at $37^{\circ} \mathrm{C}$, the reaction was started by adding NADPH followed by incubation for the appropriate time. The reaction was stopped using ice-cold acetonitrile $[100 \%(\mathrm{v} / \mathrm{v})]$ containing a different $\mathrm{AZ}$ compound as the internal standard. The mixture was centrifuged at $3000 \mathrm{~g}, 4^{\circ} \mathrm{C}$ for 10 minutes, and the supernatant was transferred to a clean 96-well plate for injection into the mass spectrometer.

Substrate Inhibition Kinetics. Linearity of formation of the metabolites M3 and M5 was established with respect to protein concentration (HLM and rCYP2C8) and time. The incubation times were 20 and 30 minutes for HLM and $\mathrm{rCYP} 2 \mathrm{C} 8$, respectively. The reaction rate with increasing concentrations of AZD9496 $(0.05-200 \mu \mathrm{M})$ was determined as described earlier (incubation conditions for recombinant human P450s and HLM). Standard curves were prepared by adding known concentrations $(5-1000 \mathrm{nM})$ of M3 and M5 to reaction mixtures (without AZD9496 or NADPH), and the samples were processed for analysis by mass spectrometry as stated earlier.

Recombinant Human P450 Screen for Formation of M3 and M5. A total of nine recombinant human P450 isoforms were screened for their contribution to formation of the major metabolites of AZD9496, M3 and M5. Each rP450 $(20 \mathrm{pmol})$ was incubated with $2 \mu \mathrm{M}$ AZD 9496 for 25 minutes at $37^{\circ} \mathrm{C}$. No standard curves were prepared, and the amounts of M3 and M5 formed were expressed as the ratio of peak area of metabolite/peak area of internal standard.

Effect of AZD9496 on Amodiaquine Deethylation. The effect of AZD9496 on amodiaquine deethylation was determined by adding AZD9496 (final concentration $100 \mu \mathrm{M}$ ) and amodiaquine (final concentration $50 \mu \mathrm{M}$ ) to the reaction mixture. Control experiments were included in which NADPH was added after stopping the reaction using ice-cold acetonitrile containing internal standard. The amount of desethylamodiaquine was quantified as the response ratio (peak area of desethylamodiaquine:peak area of internal standard).

Intrinsic Clearance of AZD9496 and Amodiaquine. Amodiaquine and AZD9496 were incubated separately at a final concentration of $1 \mu \mathrm{M}$ with human liver microsomes from eight different donors (final concentration of $1 \mathrm{mg} / \mathrm{ml}$ HLM). The reaction was started by adding NADPH, and aliquots were taken at $0,2,4,6,8$, and 10 minutes for amodiaquine and at $0,5,10,20,40$, and 60 minutes for AZD9496 and added to acetonitrile [100\% (v/v) containing internal standard]. The amount of amodiaquine or AZD9496 remaining (peak area:peak area of internal standard) at each time point was calculated as a percentage of the amount at the zero time point, and the intrinsic clearance $\left(\mathrm{CL}_{\text {int }}\right)$ was determined as described previously (Obach et al., 1997).

Metabolism of AZD9496 by Human Hepatocytes. Cryopreserved human hepatocytes (Bioreclamation IVT) were rapidly thawed in a $37^{\circ} \mathrm{C}$ water bath and added to prewarmed Lebovitz medium. After gentle mixing, the cell suspension was centrifuged at $40 \mathrm{~g}$ for 4 minutes at room temperature. After removing excess medium, the cells were resuspended in about $5 \mathrm{ml}$ of medium. An aliquot $(100 \mu \mathrm{l})$ 
of the cell suspension was added to trypan blue $[0.1 \%(\mathrm{v} / \mathrm{v})]$ solution, and the mixture was used for manual counting of cells using the improved Neubauer counting chamber. Cells with greater than $80 \%$ viability were used. Cells $(247.5 \mu \mathrm{l})$ were added to a 96-well plate and prewarmed to $37^{\circ} \mathrm{C}$ on a Microlab Star (Hamilton Robotics, Inc, Nevada). The reaction was started by adding AZD9496 $(2.5 \mu \mathrm{l})$ to achieve final concentrations $(0-200 \mu \mathrm{M})$. The final concentration of organic solvent was $0.75 \%$. An aliquot was taken after a 20 -minute incubation and added to acetonitrile $[100 \%(\mathrm{v} / \mathrm{v})$ containing internal standard]. After centrifugation, the supernatant was injected into the mass spectrometer.

Nonspecific Binding of AZD9496 to Human Liver Microsomes. Nonspecific binding of AZD9496 to human liver microsomes was assessed using the rapid equilibrium dialysis 96-well kit from Thermo Fisher Scientific (Rockford, IL). AZD9496 (at different concentrations covering those used for determining kinetic constants) was mixed with the appropriate concentration of human liver microsomes $(0.025,0.25$, or $1 \mathrm{mg} / \mathrm{ml})$ and potassium phosphate buffer $(0.1 \mathrm{M}$, $\mathrm{pH}$ 7.4). The mixture was dialyzed against buffer (potassium phosphate $0.1 \mathrm{M}$, $\mathrm{pH} 7.4$ ) at $37^{\circ} \mathrm{C}$ with shaking for 4 hours. An equal volume was taken from the buffer-only and human liver microsomal-containing chambers. A corresponding volume of human microsomal mixture was added to the buffer-only sample, and similarly, a corresponding volume of buffer was added to the sample taken from the human liver microsomal-containing chamber. After adding acetonitrile (100\% containing internal standard), the mixture was vortex-mixed and centrifuged at $3000 \mathrm{~g}$ for 10 minutes. The supernatant was transferred to a fresh 96 -well plate, and $3 \mu \mathrm{l}$ was injected into the mass spectrometer. The concentration of compound was calculated as the ratio of the peak area of compound:peak area of internal standard. The percentage free was calculated as (the concentration in buffer chamber/concentration in HLM chamber) $\times 100 \%$.

Effect of AZD9496 on CYP2C8 mRNA in HepaRG Cells. Cryopreserved HepaRG cells were thawed and plated at a density of 100,000 viable cells per well in collagen 1-coated 96-well plates as per the manufacturer's instructions. Approximately 72 hours after seeding, cells were incubated with $0.0091-20 \mu \mathrm{M}$ AZD 9496 or rifampicin (positive control inducer) or vehicle control for 48 hours, with compound solutions replaced after 24 hours. All compound solutions were prepared in Williams' medium $\mathrm{E}$ with serum-free induction supplement and a final concentration of $0.1 \%$ dimethylsulfoxide. After 48-hour treatment, cells were washed with Dulbecco's phosphate-buffered saline and then assessed for induction of P450 mRNA. mRNA was prepared from the plated cells according to the FastLane Cell Multiplex NR kit protocol, and relative expression levels were evaluated by quantitative reverse-transcription polymerase chain reaction using CYP2C8-specific primers and probes and glyceraldehyde-3-phosphate dehydrogenase as a housekeeping gene. Fold induction of CYP2C8 mRNA was calculated for each well relative to vehicle control wells and plotted against test item concentration.

Liquid Chromatography with Mass Spectrometric Detection. Chromatography of AZD9496 and its two metabolites was performed on a Waters Acquity UPLC pump (Waters, Milford, MA) fitted with a C-18 Kinetex column $(50 \times 2.1$ internal diameter, $2.6 \mu \mathrm{m})$ (Phenomenex, California) maintained at a temperature of $50^{\circ} \mathrm{C}$ with A) $0.1 \%$ formic acid in water and B) $0.1 \%$ formic acid in methanol $[100 \%(\mathrm{v} / \mathrm{v})]$ as mobile phases. The gradient elution program at a flow rate of $0.6 \mathrm{ml} / \mathrm{min}$ started with $95 \%$ A for 1.5 minutes, a decrease to $25 \%$ in 3.3 minutes, held at $25 \%$ for 0.5 minutes, back to $95 \%$ in 0.1 minutes, and held for 0.1 minutes to give a total run time of 5.5 minutes. Mass spectrometric detection was done in positive mode on the Waters TQD (Waters) with data acquisition done using MassLynx V4.1 (Waters). The gas flow was set at 600 (desolvation) and $50 \mathrm{l} / \mathrm{h}$ (cone). The desolvation and source temperatures were set at $350^{\circ} \mathrm{C}$ and $120^{\circ} \mathrm{C}$, respectively. The capillary voltage was $3.27 \mathrm{kV}$, and the analytes were monitored by multiple reaction monitoring, with the details of the scan parameters shown in Table 1.

Chromatography of amodiaquine and its metabolite desethylamodiaquine was also performed on a Waters Acquity UPLC pump fitted with a C-18 Kinetex column $(50 \times 2.1$ internal diameter, $2.6 \mu \mathrm{m})$ maintained at a temperature of $50^{\circ} \mathrm{C}$ with A) $0.1 \%$ formic acid in water and B) $0.1 \%$ formic acid in methanol $[100 \%(\mathrm{v} / \mathrm{v})]$ as mobile phases. The gradient elution program at a flow rate of $0.6 \mathrm{ml} / \mathrm{min}$ started with $95 \%$ A for 0.3 minutes, a decrease to $5 \%$ in 2.5 minutes, held at $5 \%$ for 0.2 minutes, back to $95 \%$ in 0.2 minutes, and held for 0.3 minutes to give a total run time of 3.5 minutes. Mass spectrometric detection was done in positive mode on the Waters Xevo TQS (Waters) with data acquisition performed using MassLynx V4.1 (Waters). The capillary voltage was set
TABLE 1

Mass spectrometry scan parameters

\begin{tabular}{lcccc}
\hline \multicolumn{1}{c}{ Analyte } & Parent Ion & Product Ion & Cone $(\mathrm{V})$ & Collision Energy (V) \\
\hline AZD9496 & 443.4 & 117.9 & 25 & 28 \\
M3 & 133.8 & 30 & 18 & \\
M5 & 459.4 & 133.8 & 25 & 28 \\
\hline
\end{tabular}

at $0.71 \mathrm{kV}$ and desolvation temperature was set at $600^{\circ} \mathrm{C}$. Gas flows were set as follows: desolvation $(1000 \mathrm{l} / \mathrm{h})$ and cone $(150 \mathrm{l} / \mathrm{h})$. Amodiaquine and desethylamodiaquine were monitored by multiple reaction monitoring at cone and collision voltages of 20 and $22 \mathrm{~V}$, respectively. The transitions for amodiaquine were $356.5>284.5$ and for desethylamodiaquine, $328.6>283.4$.

Data and Statistical Analysis. The inhibition data generated in this study were fitted to the generally accepted model for substrate inhibition analogous to uncompetitive inhibition (Yoshino and Murakami, 2015) (model 1) in GraphPad Prism Version 7.02 (GraphPad Software Inc., La Jolla, CA):

$$
\mathrm{V}=\mathrm{V}_{\max } /\left(\mathrm{K}_{\mathrm{m}}+[\mathrm{S}]\left(1+[\mathrm{S}] / \mathrm{K}_{\mathrm{i}}\right)\right.
$$

New models of substrate inhibition essentially similar to model 1 with the exception of an additional substrate molecule, added to try and explain the greater extent of inhibition that model 1 failed to describe, were considered. The binding of two additional molecules (forming an $\mathrm{S}_{1} \mathrm{~S}_{2} \mathrm{ES}$ complex) was assumed taking into account proposals by others (Korzekwa et al., 1998) in which some atypical reactions may involve binding of two substrate molecules and an effector molecule. Model 2 assumed sequential binding of two molecules, with the first facilitating binding of the second as shown here:

$\mathrm{E}+\mathrm{S} \underset{\mathrm{ES}}{k_{2}+} \stackrel{k_{1}}{\longrightarrow} \mathrm{E}+\mathrm{P}$

$\mathrm{S}_{1}$

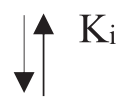

$\mathrm{S}_{1} \mathrm{ES}$

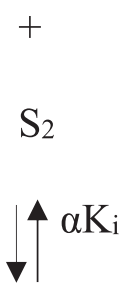

\section{$\mathrm{S}_{2} \mathrm{~S}_{1} \mathrm{ES}$}

This gives eq. 2 :

$$
\mathrm{V}=\mathrm{V}_{\max }[\mathrm{S}] /\left(\mathrm{K}_{\mathrm{m}}+[\mathrm{S}]\left(1+[\mathrm{S}] / \mathrm{k}_{\mathrm{i}}+[\mathrm{S}]^{2} / \alpha \mathrm{k}_{\mathrm{i}}^{2}\right)\right)
$$

The third model assumed simultaneous binding of $n$ (number determined from the data) molecules of AZD9496 to the inhibitor site as shown here: 

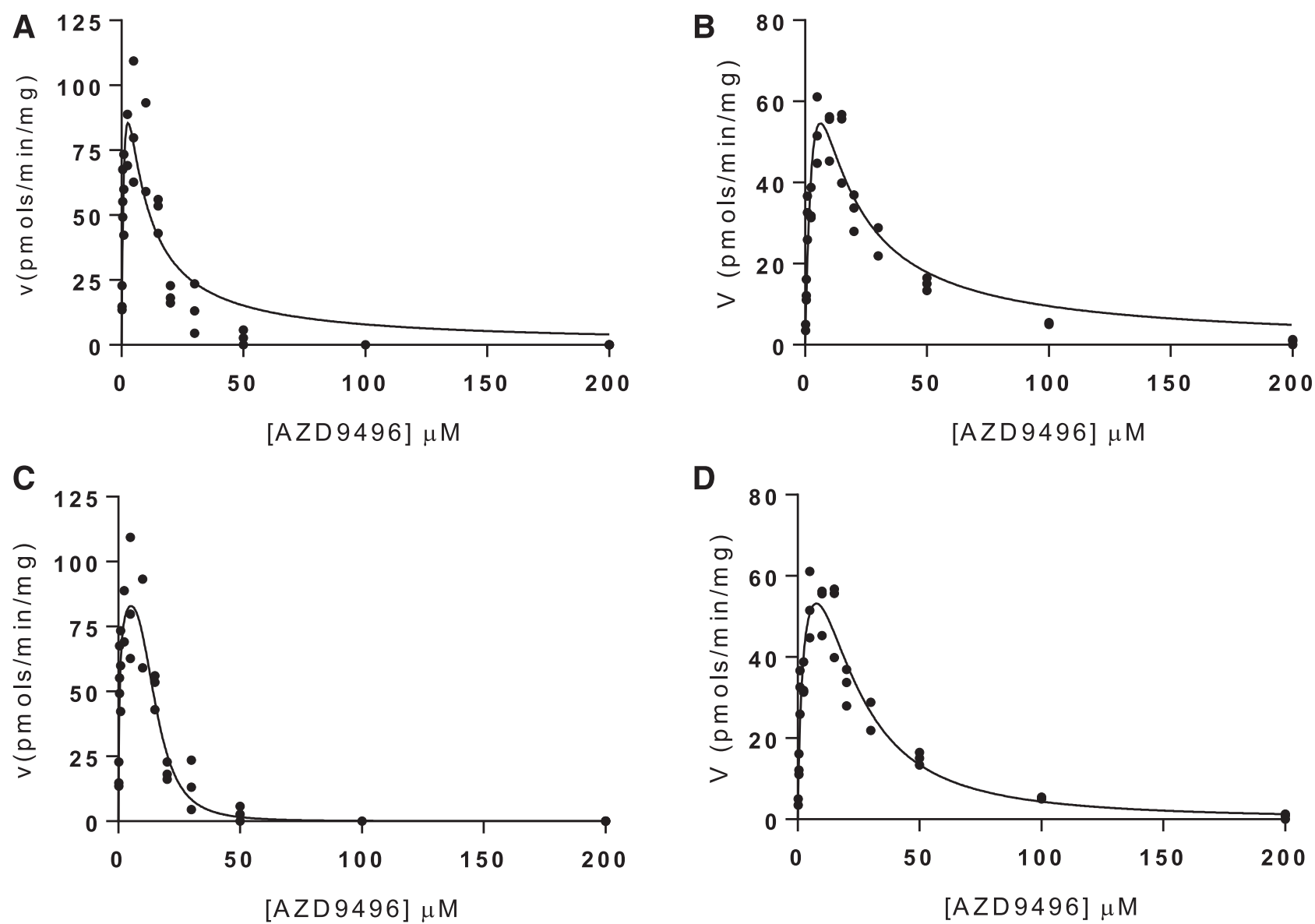

Fig. 2. The rate of formation of M3, the major metabolite of AZD9496, with increase in AZD9496 concentration in pooled HLMs at concentrations of $0.025 \mathrm{mg} / \mathrm{ml}$ (A) and $0.25 \mathrm{mg} / \mathrm{ml}$ (B) microsomal protein. Each point represents duplicate determinations, and these were repeated on three separate occasions $(n=3)$. The data in (A) and (B) were fit to the generally accepted substrate inhibition model 1. (C) and (D) were fit using the new model in this study (model 3). (C and D) Fit of the data with $0.025 \mathrm{mg} / \mathrm{ml} \mathrm{HLM}$ (C) and $0.25 \mathrm{mg} / \mathrm{ml}$ HLM (D).

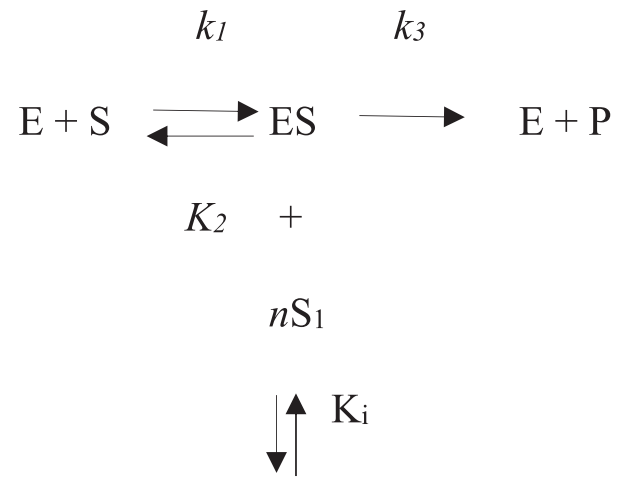

\section{$n \mathrm{~S}_{1} \mathrm{ES}$}

$$
\mathrm{V}=\mathrm{V}_{\max } /\left(\mathrm{K}_{\mathrm{m}}+[\mathrm{S}]\left(1+[\mathrm{S}]^{\mathrm{n}} / \mathrm{K}_{\mathrm{i}}^{\mathrm{n}}\right)\right)
$$

Equations 2 and 3 were imported into GraphPad Prism and were tested for their ability to describe the inhibition data in this study. Comparison of the models was done in GraphPad Prism using the extra-sum-of-squares F test. An F ratio around 1 shows the simpler model 1 to be the correct one, but a ratio much greater than 1 supports use of the more complicated one. A $P$ value can then be used to show statistical significance-a $P$ value less than 0.05 shows that the simpler model (fewer parameters, and in this study, eq. 1) is incorrect. The data and statistical analysis comply with the recommendations on experimental design and analysis in pharmacology (Motulsky and Christopoulos, 2004).

Concentration of AZD9496 Available to Interact with Enzymes In Vivo. The free concentration of AZD9496 likely to interact with enzymes in vivo after administration of a 600-mg dose was estimated according to Ito et al. $(1998,2002)$ and Rostami-Hodjegan and Tucker (2004) assuming complete solubility and bioavailability. The maximum intestinal concentration $=$ molar dose $/ 250 \mathrm{ml}$, the maximum liver inlet concentration $\left(\mathrm{I}_{\text {in } \max }\right)=\mathrm{F}_{\mathrm{a}} \cdot \mathrm{K}_{\mathrm{a}} \cdot \operatorname{dose} / \mathrm{Qh}$, and $\mathrm{I}_{\text {in free }}=$ $\mathrm{I}_{\text {in max }} \cdot \mathrm{fu} . \mathrm{I}=$ total $\mathrm{C}_{\max }$, and $\mathrm{F}_{\mathrm{a}}$ is the fraction absorbed, and this was assumed to be $1 . \mathrm{K}_{\mathrm{a}}$ is the absorption rate constant, which was assumed to be 0.1 minute $^{-1}$. $\mathrm{Qh}$ is the hepatic blood flow $(1500 \mathrm{ml} / \mathrm{min})$. A blood-to-plasma ratio of 0.66 (experimentally determined) was used, and for the fraction unbound, the Food

TABLE 2

Enzyme kinetic parameters (S.E. in parentheses) for AZD9496 in HLM with the different models

\begin{tabular}{lcr}
\hline & Equation 1 & Equation 3 \\
\hline $\mathrm{HLM} 0.025 \mathrm{mg} / \mathrm{ml}$ & & \\
$\mathrm{K}_{\mathrm{m}}(\mu \mathrm{M})$ & $1.3(1.0)$ & $0.4(0.2)$ \\
$\mathrm{V}_{\max }(\mathrm{pmol} / \mathrm{min} / \mathrm{mg})$ & $194(69)$ & $93(8.4)$ \\
Value of $n$ & 1 & $3(0.8)$ \\
$\mathrm{K}_{\mathrm{i}}(\mu \mathrm{M})$ & $3.3(2.2)$ & $12(1.4)$ \\
$\mathrm{HLM} 0.25 \mathrm{mg} / \mathrm{ml}$ & $2.5(3.7)$ & $0.8(0.7)$ \\
$\mathrm{K}_{\mathrm{m}}(\mu \mathrm{M})$ & $169(68)$ & $76(12)$ \\
$\mathrm{V}_{\max }(\mathrm{pmol} / \mathrm{min} / \mathrm{mg})$ & 1 & $1.8(0.4)$ \\
$\mathrm{V}_{\text {alue of } n}$ & $2.3(3.2)$ & $8.1(4.4)$ \\
$\mathrm{K}_{\mathrm{i}}(\mu \mathrm{M})$ & & \\
\hline
\end{tabular}




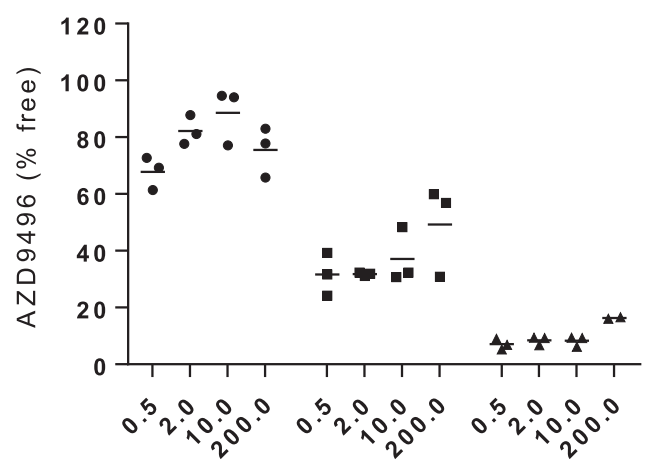

[AZD 9496] $\mu \mathrm{M}$

- $0.025 \mathrm{mg} / \mathrm{m} \mathrm{I}$

- $0.25 \mathrm{~m} \mathrm{~g} / \mathrm{m} \mathrm{I}$

$\Delta \quad 1.0 \mathrm{mg} / \mathrm{m} \mathrm{I}$

Fig. 3. The extent of nonspecific binding of AZD 9496 at different concentrations $(0.5-200 \mu \mathrm{M})$ to three different concentrations of $\operatorname{HLM}(0.025,0.25$, and $1 \mathrm{mg} / \mathrm{ml})$ measured by rapid equilibrium dialysis. A single experiment was performed at each concentration in triplicate.

and Drug Administration guidance value of 0.01 (Food and Drug Administration, 2017) for highly bound compounds was applied.

\section{Results}

Metabolism of AZD9496 by Human Liver Microsomes. The HLM protein concentrations and time that gave linear formation of the metabolites of AZD9496 were established. The effect of increasing concentrations of AZD9496 on the rate of metabolism in HLMs was investigated using 0.025 and $0.25 \mathrm{mg} / \mathrm{ml}$ of HLM protein for 20 minutes. At both 0.025 and $0.25 \mathrm{mg} / \mathrm{ml} \mathrm{HLM}$ protein, the rate of formation of the major metabolite M3 showed complete substrate inhibition (Fig. 2, A and B) - that is, the rate of formation of the metabolite went down to zero at $200 \mu \mathrm{M}$ AZD9496. The minor metabolite M5 also showed a similar pattern of substrate inhibition.

The data for formation of the major metabolite (M3) fit poorly to the generally accepted model of substrate inhibition analogous to uncompetitive inhibition (model 1), and the poor fit was more pronounced when less enzyme $(0.025 \mathrm{mg} / \mathrm{ml}$ HLM protein) was used (Fig. 2, A and B). Two additional models to the generally accepted substrate inhibition model were used to fit the data as described in Materials and Methods. Fitting the inhibition data to the second model gave visually better fits; however, these were deemed ambiguous by the program and did not generate kinetic parameters. Model 3 was a modification of model 1 that did not have any constraints on the number of [S] that could bind to the inhibitor site, i.e., while $[\mathrm{S}]$ is equal to one in model 1 , this value was determined from the data by the program for model 3. Modeling the data using eq. 3 gave much better fits (Fig. 2, C and D). In addition, the two models were compared using the extra-sum-of-squares $\mathrm{F}$ test. The $\mathrm{F}$ ratios obtained were $22(P<0.0001)$ and $10(P=0.0034)$ for 0.025 and $0.25 \mathrm{mg} / \mathrm{ml} \mathrm{HLM}$, respectively. The enzyme kinetic parameters obtained using models 1 and 3 are shown in Table 2 with $K_{m}$ and $K_{i}$ values corrected for protein binding, which is described here.

Nonspecific Protein Binding. The nonspecific binding of AZD9496 to HLM in vitro was assessed using rapid equilibrium dialysis at three different HLM concentrations of $0.025,0.25$, and $1 \mathrm{mg} / \mathrm{ml}$. The percentage of AZD9496 nonspecifically bound to HLM increased with increase in HLM protein concentration. At least 91\% of AZD9496 was nonspecifically bound to the HLM matrix at the highest HLM concentration of $1 \mathrm{mg} / \mathrm{ml}$ (Fig. 3). At the four concentrations of AZD9496 tested $(0.5-200 \mu \mathrm{M})$, there did not seem to be a significant difference in the extent of nonspecific binding at each HLM concentration except at the $1.0 \mathrm{mg} / \mathrm{ml}$ HLM concentration, where $200 \mu \mathrm{M}$ AZD9496 seemed to result in a higher percentage of free drug ( $16 \%$ compared with about $7 \%$ at the other concentrations).

P450 Identification. A total of nine recombinant human P450 isoforms were screened for their contribution to formation of the main metabolites of AZD9496, M3 and M5. The concentrations of M3 and M5 were expressed as the response ratio (peak area of metabolite:peak area of internal standard). M3 and M5 were detected in incubations containing CYP2C8 only (Fig. 4A). To confirm CYP2C8 as the major isoform responsible for metabolism of AZD9496, a correlation analysis of the $\mathrm{CL}_{\text {int }}$ of AZD9496 and the CYP2C8-specific probe amodiaquine was performed in HLMs from eight different donors. A good correlation $\left(r^{2}=0.78\right)$ was observed between $\mathrm{CL}_{\text {int }}$ values for AZD9496 and amodiaquine (Fig. 4B).
A

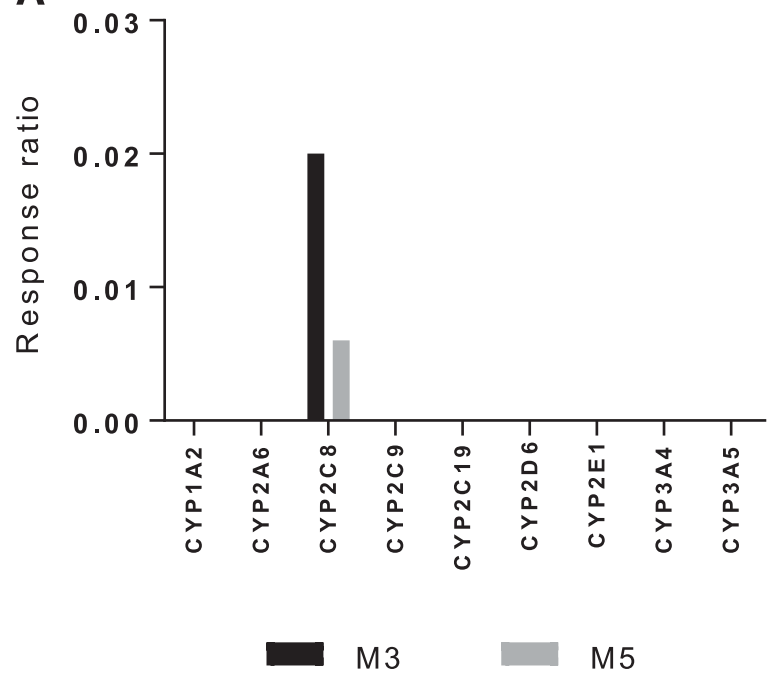

B

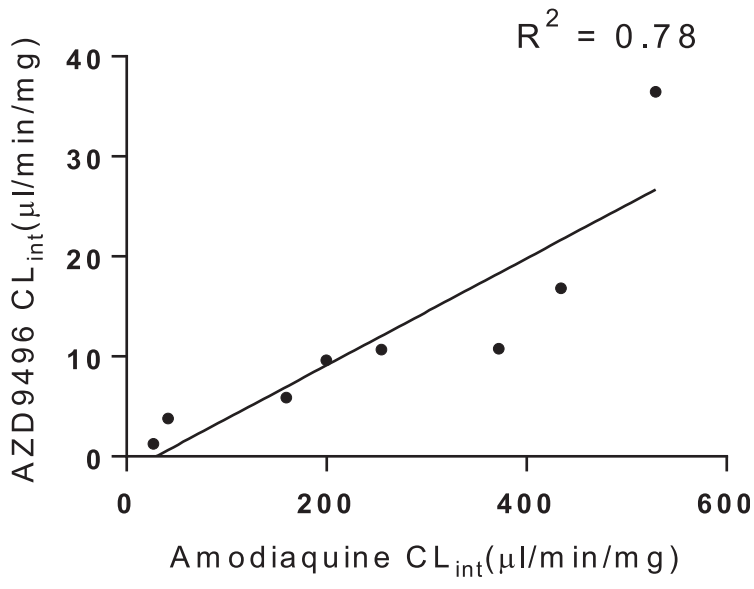

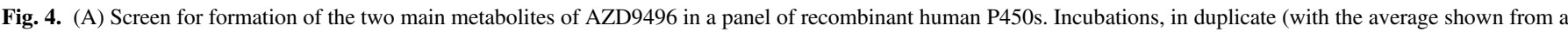

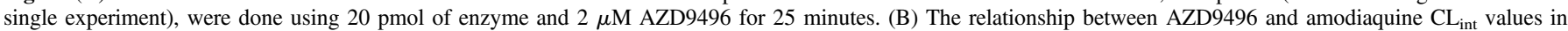
human liver microsomes from eight different donors. The results are the average of duplicate determinations repeated on three separate occasions $(n=3)$. 


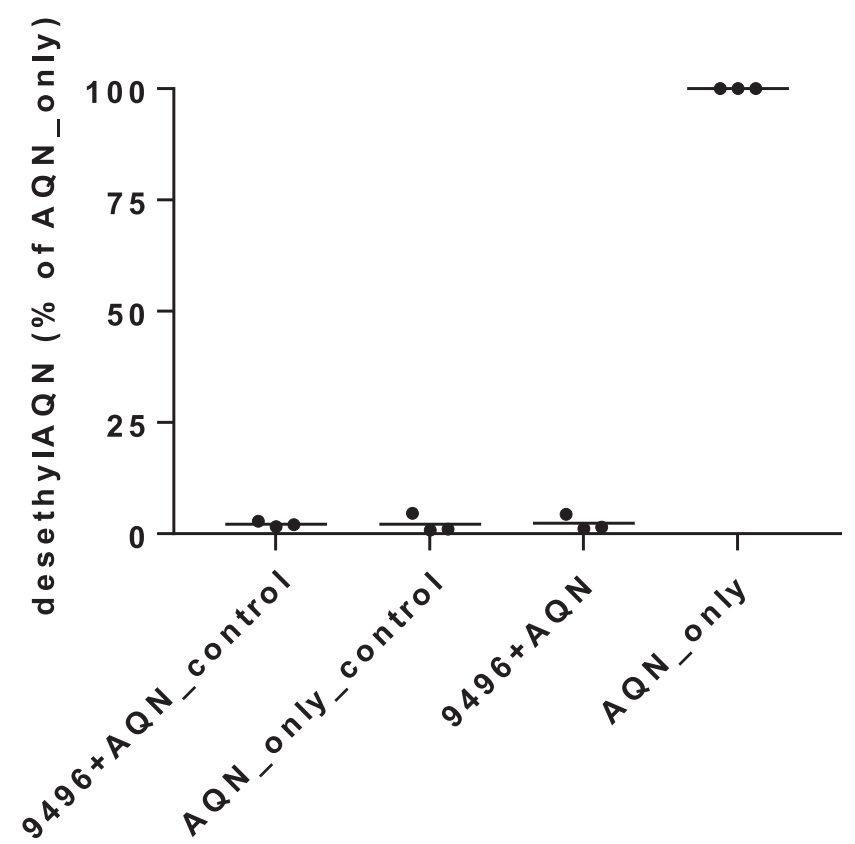

Fig. 5. The effect of AZD9496 $(100 \mu \mathrm{M})$ on formation of desethylamodiaquine (measured as a percentage, with the amodiaquine-only incubation set at 100\%) after incubation of amodiaquine $(50 \mu \mathrm{M})$ with $0.025 \mathrm{mg} / \mathrm{ml}$ of human liver microsomes for 20 minutes. The reaction in the samples was started by adding NADPH, whereas NADPH was added to the controls after stopping the reaction with ice-cold acetonitrile. Each point represents the average of triplicate determinations, and these were done on three separate occasions $(n=3)$. AQN, amodiaquine.

Completeness of Substrate Inhibition. To check the completeness of the substrate inhibition and to rule out the possibility of AZD9496specific artifacts, the impact of AZD9496 on the formation of desethylamodiaquine from amodiaquine in HLM was assessed. The concentration of amodiaquine used was $50 \mu \mathrm{M}$ while AZD9496 was incubated at $100 \mu \mathrm{M}$ (the same concentration that inhibited $100 \%$ of M3 formation at $0.025 \mathrm{mg} / \mathrm{ml} \mathrm{HLM}$ protein). The amount of desethylamodiaquine formed was quantified by measuring the peak area to internal standard ratio, and this was expressed as a percentage with the amodiaquine only incubation set at $100 \%$. NADPH (the cofactor required by P450s) was added to the control incubations for amodiaquine only and amodiaquine plus AZD9496 after stopping the reaction. These were compared with the reactions in which NADPH was present at the start. The controls had levels of desethylamodiaquine similar to the AZD9496 plus amodiaquine incubation, showing that AZD9496 caused complete inhibition of desethylamodiaquine formation (Fig. 5).

Significance of the Value of $\boldsymbol{n}$. Given the different numbers of $n$ (number of AZD9496 molecules bound to the inhibitor site) predicted by GraphPad Prism under different conditions (protein concentration), the effect of enzyme concentration on the value of $n$ was studied using recombinant CYP2C8 (0.4 and 4 pmol). Given the extensive nonspecific protein binding of AZD9496, the protein concentration was kept constant by adding control baculovirus microsomes (heterologous expression system used to produce the recombinant human CYP2C8 used in this study). The number of AZD9496 molecules predicted to bind to the inhibitor site was 2-fold higher when less enzyme was used (Fig. 6). The $\mathrm{K}_{\mathrm{m}}$ values were the same $(0.67 \mu \mathrm{M})$, but the $\mathrm{K}_{\mathrm{i}}$ value was higher when less enzyme was used (14 compared with $6 \mu \mathrm{M})$.

Confirmation of Substrate Inhibition in Human Hepatocytes. Human hepatocytes were used to assess whether the substrate inhibition exhibited by AZD9496 in HLM and rCYP2C8 was not a microsomal artifact. Human hepatocytes at 0.125 and 1 million cells $/ \mathrm{ml}$ were incubated with increasing concentrations of AZD9496. A profile of substrate inhibition similar to that in HLM and recombinant CYP2C8 was observed in hepatocytes with almost complete inhibition observed when fewer cells were used (Fig. 7). Multiple AZD9496 molecules may also bind to the enzymes in hepatocytes as shown by $n$ values of 2.2 and 3 for 0.125 and 1 million cells $/ \mathrm{ml}$, respectively. Similar $K_{m}$ values of 7.8 and $8.7 \mu \mathrm{M}$ were observed.

Induction of CYP2C8 mRNA in HepaRG Cells. Plated HepaRG cells were used to assess whether AZD9496 induced CYP2C8 mRNA (Fig. 8). The magnitude of CYP2C8 mRNA induction in plated HepaRG cells was observed to be small, with the positive control inducer rifampicin demonstrating a calculated $\mathrm{E}_{\max }$ of $1.8 \pm 0.13$-fold. AZD9496 also demonstrated a concentration-dependent induction of CYP2C8 mRNA to a maximum at $20 \mu \mathrm{M}$ that was $155 \%$ of the rifampicin control.

Free AZD9496 Concentrations Achieved after Administration of a Single 600-mg Dose. After administration of a single 600-mg dose of AZD9496, the total plasma $\mathrm{C}_{\max }$ was $17.9 \mu \mathrm{M}$ (Hamilton et al., 2018). This gave a predicted intestinal concentration of $5424 \mu \mathrm{M}$, and $\mathrm{I}_{\text {in max }}$ of $102 \mu \mathrm{M}$. The $\mathrm{I}_{\text {in free }}$, estimated using the Food and Drug Administration guidance for highly bound compounds (fu of 0.01), was $1.0 \mu \mathrm{M}$.

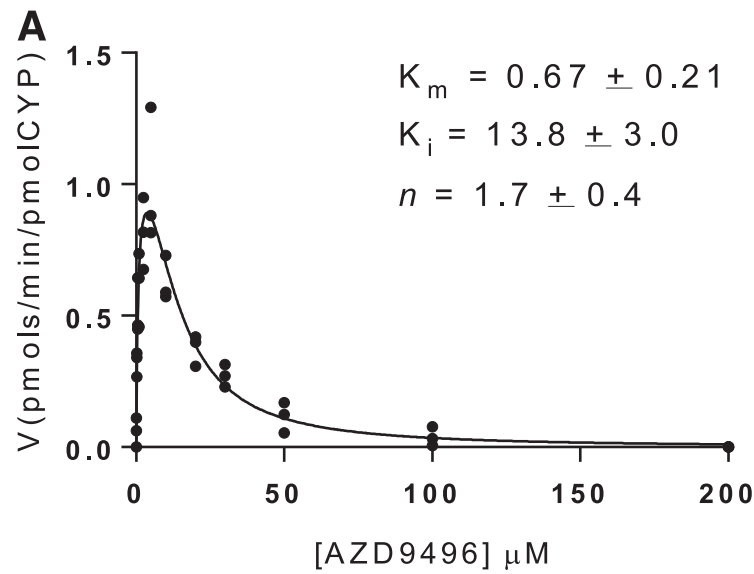

B

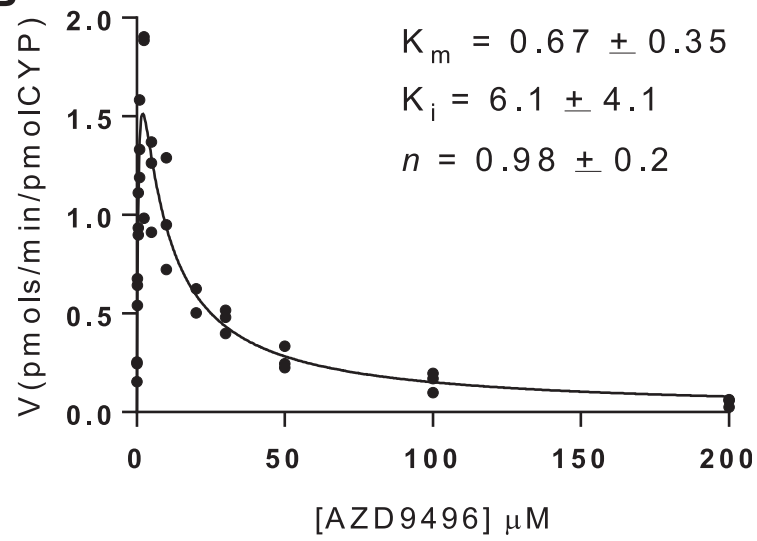

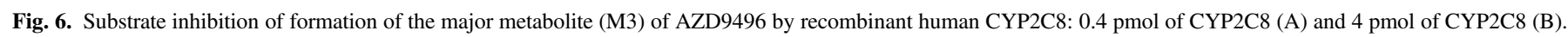

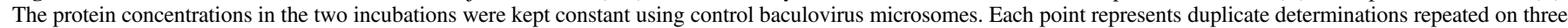
separate occasions $(n=3)$. 
A

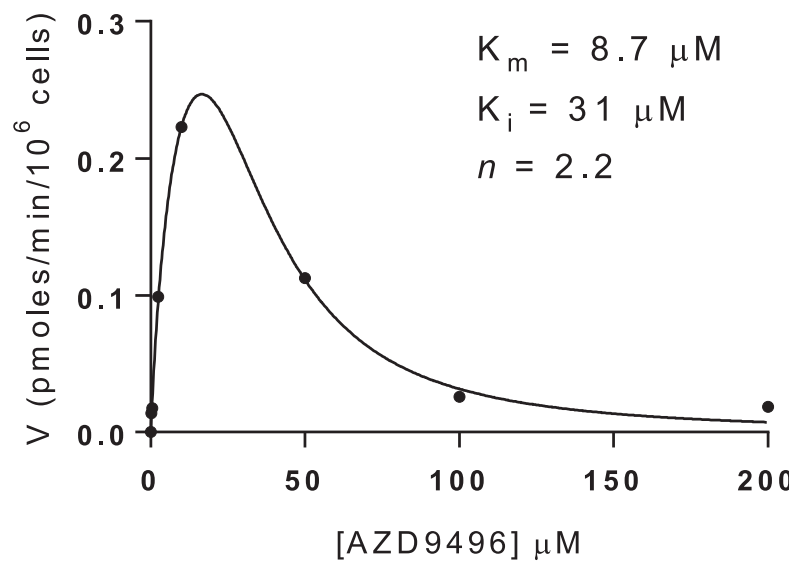

B

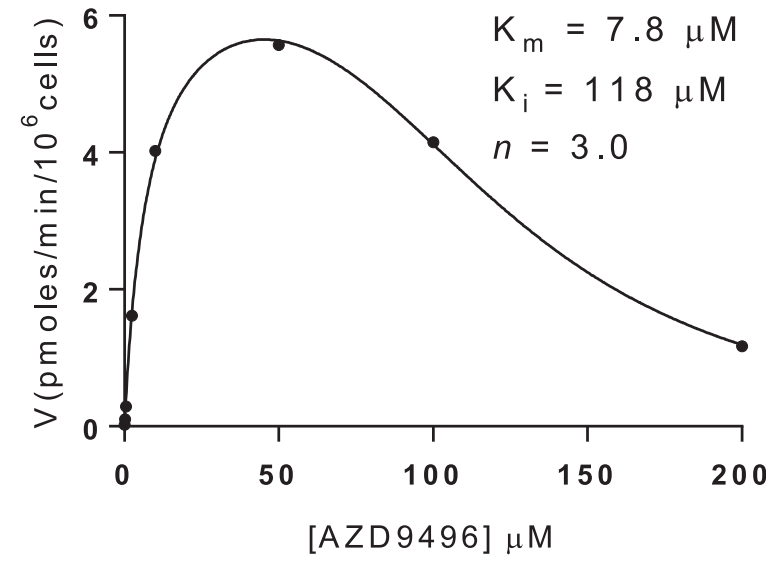

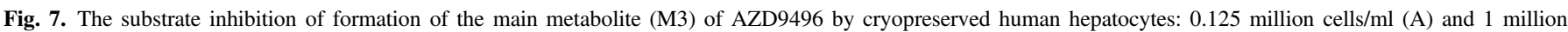
cells $/ \mathrm{ml}$ (B). The results are the average of duplicate determinations from a single experiment.

\section{Discussion}

Substrate inhibition is a common mechanism of enzyme inhibition; however, many questions have been raised concerning its relevance. Indeed, some have dismissed it as an in vitro artifact (Reed et al., 2010), as the majority of cases of substrate inhibition occur at very high substrate concentrations that would almost never be achieved in vivo (Lin et al., 2001). In addition to an in vitro microsomal system, others have suggested the use of other systems, such as hepatocytes, to confirm any observed substrate inhibition (Houston and Galetin, 2005). In this study, HLM, recombinant human CYP2C8, and human hepatocytes were used to show a similar profile of complete substrate inhibition of CYP2C 8 by the oral selective estrogen receptor degrader AZD9496. In addition, the completeness of the inhibition was demonstrated using the specific probe of CYP2C8 activity amodiaquine deethylation.

The substrate inhibition observed with AZD9496 did not conform to the generally accepted model analogous to uncompetitive inhibition, model 1 (Yoshino and Murakami, 2015), with the data showing a greater degree of inhibition than could be described by the model. Additionally, visual inspection of the data and kinetic parameters showed that this was not the correct model to use- - the $\mathrm{V}_{\max }$ values were much higher than was suggested by the data. Mathematically, one of the ways in which the greater degree of inhibition could be accounted for was an increase in the number of substrate molecules causing the inhibition, i.e., increasing the value of $[\mathrm{S}]$ in the denominator of eq. 1. The fitting of two or more substrate molecules in the active site that could theoretically include an inhibitory site is supported by the large volume of the CYP2C8 active site (Schoch et al., 2004). Additional support for multiple substrate binding comes from studies by others which show that a substrate can adopt multiple binding orientations within the CYP2C8 active site (Kerdpin et al., 2004). Therefore, it is possible for at least two molecules of AZD9496 to bind to the active site of CYP2C8 in productive and nonproductive orientations. Inhibition of the enzyme could theoretically occur because of a molecule bound in a nonproductive orientation interfering with the productive binding, thus reducing the rate of reaction or preventing the product from leaving the active site.

Model 2 was derived based on the binding of two substrate molecules to an inhibitor site, with the first molecule facilitating binding of the next (eq. 2). Visually, the model gave a better fit compared with the generally accepted one (model 1); however, the program could not give values of the parameters, as this was deemed an ambiguous fit. The generally accepted model 1 was modified to give model 3 by removing any constraints on the number of AZD9496 molecules occupying the inhibitor site, with the number of [S] determined by the inhibition data. Notably, model 3 gave the best fits of the data by assigning different numbers of molecules bound to the inhibitory site based on the data. In addition, comparison of the two models using the sum-of-squares $\mathrm{F}$ test and associated $P$ values showed model 3 to be the better model. The number $(n)$ of molecules of AZD9496 predicted to interact with the inhibitor site differed depending on the amount of protein used, and this was investigated further.

AZD9496 is extensively nonspecifically bound to protein in vitro. Using different amounts of protein in incubations would, in turn, mean different concentrations of AZD9496 are available to interact with the enzyme. There was up to a 10 -fold difference in percentage free AZD9496 at $0.025 \mathrm{mg} / \mathrm{ml}$ compared with $1 \mathrm{mg} / \mathrm{ml}$ HLM protein. In addition, the difference in protein concentration would also mean a difference in the amount of enzyme. Given that the impact of protein concentration was straightforward, i.e., it reduced the concentration of AZD9496 available to interact with the enzyme, we investigated the impact of different enzyme concentrations on the enzyme kinetics using recombinant CYP2C8 (at 0.4 and 4 pmol). Microsomes from control insect baculovirus (the heterologous expression system of the human recombinant $\mathrm{CYP} 2 \mathrm{C} 8$ used in the study) were used to maintain the same

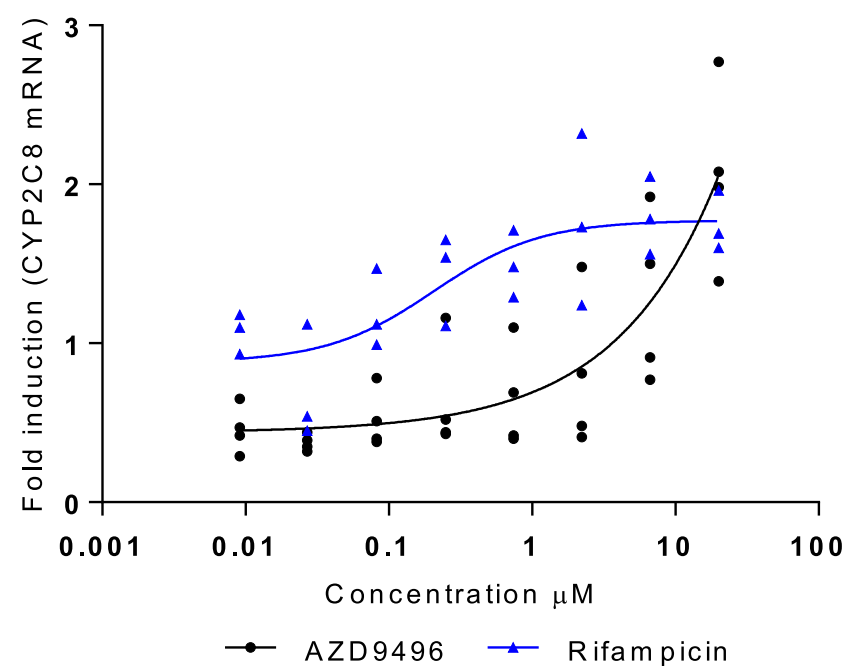

Fig. 8. Fold induction of CYP2C8 mRNA in cultured HepaRG cells by AZD9496 and rifampicin. Each data point represents an individual determination in a single experiment. 
protein concentration in both the 0.4- and 4-pmol arms of the experiment. Interestingly, the number of molecules of AZD9496 predicted to bind to the inhibitor site was higher when less enzyme was used. The higher $K_{i}$ value associated with less enzyme might seem counterintuitive but may simply reflect the binding of twice the number of AZD9496 molecules and is not an indicator of the strength of binding when comparing the two protein concentrations. The greater degree of inhibition when less enzyme is used likely comes from a "removal" of the extra molecule through binding to the inhibitor site instead of contributing to the increased rate of product formation that would occur when a higher concentration of enzyme is used. Our hypothesis is that AZD9496 initially binds to the active site in a productive orientation, with involvement of an inhibitory site occurring as more substrate becomes available. A high [S]:[E] ratio allows for the maximum number of AZD9496 molecules to occupy the inhibitor site and is supported by the higher $\mathrm{K}_{\mathrm{i}}$ values compared with $\mathrm{K}_{\mathrm{m}}$ values.

The possible in vivo relevance of the complete substrate inhibition observed in this study is intriguing. The potential for dose nonlinearity from typical M-M kinetics can be assessed by comparing the $\mathrm{K}_{\mathrm{m}}$ value to the expected in vivo free drug concentrations (Ludden, 1991). A lower $\mathrm{K}_{\mathrm{m}}$ value compared with drug concentrations would lead to saturation of metabolism and dose nonlinearity. Another possible cause of dosenonlinearity is time-dependent inhibition; however, AZD9496 did not inhibit CYP2C8 in a time-dependent manner (data not shown). For complete substrate inhibition, an increase in concentration of substrate would be expected to result in an increase in the percentage inhibition of its own metabolism across all concentrations up to $\mathrm{V}_{\max }$. Each increase in dose would, therefore, be expected to result in a higher than linear exposure, with the greatest nonlinear exposure occurring at concentrations where the rate of product formation goes down to zero. Added to this is the possible impact of the amount of CYP2C8 enzyme an individual has on the extent of inhibition, with individuals expressing highest protein likely to experience the least amount of inhibition. A wide range in extent of inhibition by AZD9496 is, therefore, expected, as CYP2C8 is polymorphic and has been shown to exhibit high interindividual variation in protein expression of up to 33 -fold (Naraharisetti et al., 2010). In the phase 1 study by Hamilton et al. (2018), single doses of AZD9496 from 20 to $400 \mathrm{mg}$ exhibited reasonably proportional increases in AUC and $\mathrm{C}_{\max }$. However, a more than doseproportional increase in $\mathrm{AUC}$ and $\mathrm{C}_{\max }$ was observed after administration of the highest single dose (600 mg on day 1). After the single 600-mg dose, the maximum unbound liver inlet concentration was estimated to be $1 \mu \mathrm{M}$, which would be higher than the $\mathrm{K}_{\mathrm{m}}$ of $0.4 \mu \mathrm{M}$ (in HLM) for CYP2C8. The extent of substrate inhibition likely to occur at this dose is not clear but could explain the apparent dose nonlinearity. Whereas formation of M3 and M5 was mediated mainly by CYP2C8, depletion of AZD9496 was observed in incubations with CYP3A4 and CYP3A5 (data not shown). Upon multiple 600-mg twice-daily dosing, the observed more-than-dose-proportional decrease in AUC on day 15 versus day 1 would be consistent with induction of CYP3A and CYP2C8 at these concentrations.

In conclusion and in contrast to previous reports (Lin et al., 2001), substrate inhibition of CYP2C8 by AZD9496 was shown to be complete and not just partial. The inhibition likely involves binding of at least two molecules of AZD9496 to the inhibitor site, which may be in the active site of the enzyme. Given that CYP2C8 is the major isoform catalyzing formation of the two major metabolites of AZD9496, our results suggest substrate inhibition as the cause of the observed dose nonlinearity at the highest single dose of AZD9496 tested in the clinic (i.e., $600 \mathrm{mg}$ ).

\section{Acknowledgments}

We thank Dr. Dermot McGinnity for useful discussions.

\section{Authorship Contributions}

Participated in research design: Bapiro, Sykes, Jones.

Conducted experiments: Bapiro, Rollison.

Performed data analysis: Bapiro, Sykes, Martin, Davies, Yates, Hoch, Rollison, Jones.

Wrote or contributed to the writing of the manuscript: Bapiro, Sykes, Martin, Davies, Yates, Hoch, Rollison, Jones.

\section{References}

Atkins WM (2005) Non-Michaelis-Menten kinetics in cytochrome P450-catalyzed reactions. Annu Rev Pharmacol Toxicol 45:291-310.

De Savi C, Bradbury RH, Rabow AA, Norman RA, de Almeida C, Andrews DM, Ballard P, Buttar D, Callis RJ, Currie GS, et al. (2015) Optimization of a novel binding motif to (E)-3-(3,5difluoro-4-((1R,3R)-2-(2-fluoro-2-methylpropyl)-3-methyl-2,3,4,9-tetrahydro-1H-pyrido[3,4-b]indol1-yl)phenyl)acrylic acid (AZD9496), a potent and orally bioavailable selective estrogen receptor downregulator and antagonist. $J$ Med Chem 58:8128-8140.

Food and Drug Administration (2017) In vitro metabolism- and transporter-mediated drug-drug interaction studies guidance for industry, Food and Drug Administration, Silver Spring, MD

Gay SC, Roberts AG, and Halpert JR (2010) Structural features of cytochromes P450 and ligands that affect drug metabolism as revealed by X-ray crystallography and NMR. Future Med Chem 2:1451-1468.

Hamilton EP, Patel MR, Armstrong AC, Baird RD, Jhaveri K, Hoch M, Klinowska T, Lindemann JPO, Morgan S, Schiavon G, et al. (2018) A first-in-human study of the new oral selective estrogen receptor degrader AZD9496 for $\mathrm{ER}^{+} / \mathrm{HER}^{-}$advanced breast cancer. Clin Cancer Res DOI: 10.1158/1078-0432.CCR-17-3102 [published ahead of print].

Houston JB (1994) Relevance of in vitro kinetic parameters to in vivo metabolism of xenobiotics. Toxicol In Vitro 8:507-512.

Houston JB and Galetin A (2005) Modelling atypical CYP3A4 kinetics: principles and pragmatism. Arch Biochem Biophys 433:351-360.

Ito K, Chiba K, Horikawa M, Ishigami M, Mizuno N, Aoki J, Gotoh Y, Iwatsubo T, Kanamitsu S, Kato M, et al. (2002) Which concentration of the inhibitor should be used to predict in vivo drug interactions from in vitro data? AAPS PharmSci 4:E25.

Ito K, Iwatsubo T, Kanamitsu S, Ueda K, Suzuki H, and Sugiyama Y (1998) Prediction of pharmacokinetic alterations caused by drug-drug interactions: metabolic interaction in the liver. Pharmacol Rev 50:387-412.

Kerdpin O, Elliot DJ, Boye SL, Birkett DJ, Yoovathaworn K, and Miners JO (2004) Differential contribution of active site residues in substrate recognition sites 1 and 5 to cytochrome P450 2C8 substrate selectivity and regioselectivity. Biochemistry 43:7834-7842.

Korzekwa KR, Krishnamachary N, Shou M, Ogai A, Parise RA, Rettie AE, Gonzalez FJ, and Tracy TS (1998) Evaluation of atypical cytochrome P450 kinetics with two-substrate models: evidence that multiple substrates can simultaneously bind to cytochrome P450 active sites. Biochemistry 37:4137-4147.

Li XQ, Björkman A, Andersson TB, Ridderström M, and Masimirembwa CM (2002) Amodiaquine clearance and its metabolism to $\mathrm{N}$-desethylamodiaquine is mediated by CYP2C8: a new high affinity and turnover enzyme-specific probe substrate. J Pharmacol Exp Ther 300: 399-407.

Lin Y, Lu P, Tang C, Mei Q, Sandig G, Rodrigues AD, Rushmore TH, and Shou M (2001) Substrate inhibition kinetics for cytochrome P450-catalyzed reactions. Drug Metab Dispos 29 : 368-374.

Ludden TM (1991) Nonlinear pharmacokinetics: clinical implications. Clin Pharmacokinet 20: 429-446.

Ma Y, Fu Y, Khojasteh SC, Dalvie D, and Zhang D (2017) Glucuronides as potential anionic substrates of human cytochrome P450 2C8 (CYP2C8). J Med Chem 60:8691-8705.

Motulsky H and Christopoulos A (2004) Fitting Models to Biological Data Using Linear and Nonlinear Regression. A Practical Guide to Curve Fitting, Oxford University Press, New York

Naraharisetti SB, Lin YS, Rieder MJ, Marciante KD, Psaty BM, Thummel KE, and Totah RA (2010) Human liver expression of CYP2C8: gender, age, and genotype effects. Drug Metab Dispos 38:889-893.

Obach RS, Baxter JG, Liston TE, Silber BM, Jones BC, MacIntyre F, Rance DJ, and Wastall P (1997) The prediction of human pharmacokinetic parameters from preclinical and in vitro metabolism data. J Pharmacol Exp Ther 283:46-58.

Obach RS and Reed-Hagen AE (2002) Measurement of Michaelis constants for cytochrome P450-mediated biotransformation reactions using a substrate depletion approach. Drug Metab Dispos 30:831-837

Pelkonen O, Turpeinen M, Uusitalo J, Rautio A, and Raunio H (2005) Prediction of drug metabolism and interactions on the basis of in vitro investigations. Basic Clin Pharmacol Toxicol 96:167-175.

Reed MC, Lieb A, and Nijhout HF (2010) The biological significance of substrate inhibition: a mechanism with diverse functions. BioEssays 32:422-429.

Rendic S and Guengerich FP (2015) Survey of human oxidoreductases and cytochrome P450 enzymes involved in the metabolism of xenobiotic and natural chemicals. Chem Res Toxicol 28: $38-42$.

Rostami-Hodjegan A and Tucker G (2004) 'In silico' simulations to assess the 'in vivo' consequences of 'in vitro' metabolic drug-drug interactions. Drug Discov Today Technol 1:441-448

Schoch GA, Yano JK, Sansen S, Dansette PM, Stout CD, and Johnson EF (2008) Determinants of cytochrome $\mathrm{P} 4502 \mathrm{C} 8$ substrate binding: structures of complexes with montelukast, troglitazone, felodipine, and 9-cis-retinoic acid. J Biol Chem 283:17227-17237.

Schoch GA, Yano JK, Wester MR, Griffin KJ, Stout CD, and Johnson EF (2004) Structure of human microsomal cytochrome P450 2C8. Evidence for a peripheral fatty acid binding site. J Biol Chem 279:9497-9503.

Spracklin DK, Hankins DC, Fisher JM, Thummel KE, and Kharasch ED (1997) Cytochrome P450 $2 \mathrm{E} 1$ is the principal catalyst of human oxidative halothane metabolism in vitro. J Pharmacol Exp Ther 281:400-411.

Tang C, Shou M, Mei Q, Rushmore TH, and Rodrigues AD (2000) Major role of human liver microsomal cytochrome P450 2C9 (CYP2C9) in the oxidative metabolism of celecoxib, a novel cyclooxygenase-II inhibitor. J Pharmacol Exp Ther 293:453-459. 
Torimoto N, Ishii I, Hata M, Nakamura H, Imada H, Ariyoshi N, Ohmori S, Igarashi T, and Kitada M (2003) Direct interaction between substrates and endogenous steroids in the active site may change the activity of cytochrome P450 3A4. Biochemistry 42:15068-15077.

Totah RA and Rettie AE (2005) Cytochrome P450 2C8: substrates, inhibitors, pharmacogenetics, and clinical relevance. Clin Pharmacol Ther 77:341-352.

Venkatakrishnan K, Von Moltke LL, and Greenblatt DJ (2001) Human drug metabolism and the cytochromes P450: application and relevance of in vitro models. J Clin Pharmacol 41: $1149-1179$.

Weir HM, Bradbury RH, Lawson M, Rabow AA, Buttar D, Callis RJ, Curwen JO, de Almeida C, Ballard P, Hulse M, et al. (2016) AZD9496: an oral estrogen receptor inhibitor that blocks the growth of ER-positive and ESR1-mutant breast tumors in preclinical models. Cancer Res $\mathbf{7 6}$ : $3307-3318$.
Williams PA, Cosme J, Vinkovic DM, Ward A, Angove HC, Day PJ, Vonrhein C, Tickle IJ, and Jhoti H (2004) Crystal structures of human cytochrome P450 3A4 bound to metyrapone and progesterone. Science 305:683-686.

Yoshino M and Murakami K (2015) Analysis of the substrate inhibition of complete and partial types. Springerplus 4:292.

Address correspondence to: Dr. Tashinga E. Bapiro, Oncology IMED Biotech Unit, AstraZeneca, Hodgkin Building c/o B310, Cambridge Science Park, Milton Road, CB4 OWG Cambridge, UK. E-mail: tashinga.bapiro1@astrazeneca.com 